small numbers. It is perhaps debatable whether hypothyroidism should be classed as a congenital malformation. If this case is excluded then the figures are $\chi^{2}=7 \cdot 98, P=0.005$.

Our data on head circumferences have been misinterpreted by Campbell and Sullivan. We did not claim that there was any statistical difference in head circumference of babies of non-drinkers compared with those of all drinkers. But there was a statistical difference when comparing the infants of mothers drinking over $20 \mathrm{ml}$ alcohol daily at the gestational ages stated. The small numbers do invite caution in interpretation of the results, and this we expressed in the discussion in our paper.

We do not claim that there is a proved association between alcohol consumption and congenital malformation, but our data make us highly suspicious that this may be so. Similar studies of larger numbers of pregnancies are required.

\section{Plasma 25-hydroxyvitamin D and rickets in low birthweight babies}

Sir,

Dr McIntosh and his colleagues have recently drawn attention to the high incidence of rickets in babies weighing $<1 \mathrm{~kg}$ at birth. ${ }^{1}$ Eight of the 15 survivors in their unit in 1981 developed radiological rickets at 2-3 months when fed on breast milk, even though they were given 2000 units of vitamin $D$ a day. They conclude that lack of vitamin $D$ is unlikely to be the cause of these bone changes, and comment on the possible role of calcium and phosphate deficiency. We were surprised, therefore, that Dr McIntosh ${ }^{1}$ and Dr Brooke ${ }^{2}$ have not commented on the evidence in favour of calcium supplements published by Day et al $^{3}$ in 1975 and Steichen $e$ al $^{4}$ in 1980.

We have not seen metaphyseal cupping of the type described by McIntosh in this hospital in the past 6 years, but we have had pathological fractures in 5 babies associated with marked osteoporosis ${ }^{5} 6$ to 9 weeks after birth. All babies of birthweight $<1 \mathrm{~kg}$ in this hospital have been fed on Gold Cap SMA since 1977 and all have had a daily supplement of 400 units of vitamin $D$. Four of the 10 survivors born between 1977 and 1980 suffered multiple pathological rib fractures on this diet, and 2 of these babies also had limb fractures that healed with appreciable angulation.

Because of this additional oral calcium supplements were introduced at the end of 1980 (total calcium intake $26 \mathrm{mmol} / 100 \mathrm{ml}$ of milk). There have been no other changes in feeding policy in the past 6 years. We have not seen a single pathological fracture in any of the 10 very low birthweight survivors born since then. Nor have we detected any radiological or biochemical evidence of rickets. While there must be a $10 \%$ possibility that this change occurred by chance, it has certainly encouraged us to continue offering calcium supplements. It would be valuable to have these findings confirmed by another unit that cares for more of these babies.

It should perhaps be pointed out that there is only 1 commercial milk preparation on sale in the United Kingdom that provides as much calcium as was used in the experimental studies of Day and Steichen, and that this contains too little sodium to be entirely satisfactory for babies of very low birthweight. We therefore use the same experimental milk formula for babies of $<1.3 \mathrm{~kg}$ as was used in the studies reported by Day et $a l^{3}$ and make this up as required from SMA milk concentrate and a mineral concentrate provided by the hospital pharmacy.

\section{References}

1 McIntosh N, Livesey A, Brooke OG. Plasma 25-hydroxyvitamin $D$ and rickets in infants of extremely low birthweight. Arch Dis Child 1982;57: 848-50.

2 Brooke OG. Low birth weight babies. Nutrition and feeding. Br J Hosp Med 1982; 28 : 462-9.

3 Day GM, Chance GW, Radde IC, Reilly BJ, Park E, Sheepers J. Growth and mineral metabolism in very low birth weight infants. II. Effects of calcium supplementation on growth and divalent cations. Pediatr Res 1975; 9: 568-75.

4 Steichen JJ, Gratton TL, Tsang RC. Osteopenia of prematurity: the cause and possible treatment. J Pediatr 1980; 96: 528-34.

5 Oppenheimer SJ, Snodgrass GJAI. Neonatal rickets. Histopathology and quantitative bone changes. Arch Dis Child 1980; 55: 945-9.

Caroline McCowen and Edmund Hey Princess Mary Maternity Hospital, Newcastle upon Tyne NE2 3BD

\section{Dr McIntosh and co-workers comment:}

The conclusion of our paper on plasma 25-hydroxyvitamin $D^{1}$ in rickets in infants less than $1000 \mathrm{~g}$ was indeed that substrate insufficiency was the likely cause. We are aware of the data of Day et $a l^{2}$ and of Steichen et $a l^{3}$ suggesting that calcium insufficiency is important, but are also aware of the case reported by Rowe $e t$ al of a breast fed infant who responded to additional phosphorus intake. ${ }^{4}$ We think that whether rickets of prematurity is due to calcium or phosphorus deficiency, or both, is still open to question. Our concern in our paper was to disprove that it was due to insufficiency of vitamin $D$.

In our paper we stated that 8 of the 15 survivors of $<1000 \mathrm{~g}$ birthweight over a 1 year period developed radiological rickets. We did no formal balance studies on these infants, but if the enteral intakes of calcium and phosphorus in the 8 babies developing rickets are compared with those of the 7 babies who did not, there is a significantly lower intake of both these minerals in the group developing rickets at 27 and 28 weeks' postconception (see Figs. 1 and 2). Allowing for the enteral fat malabsorption in infants of this gestational age, the accumulation rate for both calcium and phosphorus must be orders of magnitude less than the in utero accumulation rate given by Shaw. ${ }^{5}$ We are embarking on more formal balance studies on this extremely high risk group of babies that we would still prefer to feed on breast milk. We propose, like McCowan and Hey, to add calcium and also phosphate as Senterre and his associates (personal communication, 1983) have shown that the addition of calcium alone predisposes to nephrocalcinosis. 


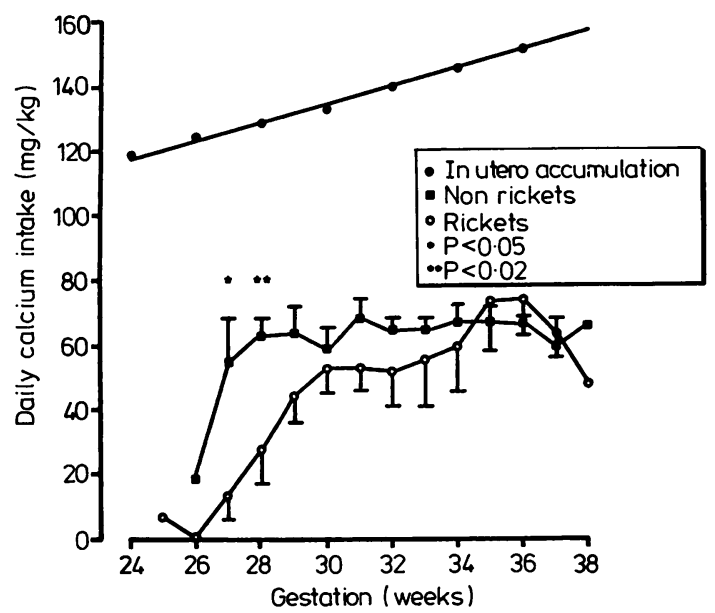

Fig. 1 Daily enteral calcium intake of 8 neonatal survivors of $<1000 \mathrm{~g}$ birthweight developing rickets (O) compared to 7 neonatal survivors of $<1000 \mathrm{~g}$ birthweight not developing rickets $(\square)$. The in utero accumulation rate of Shaw is also shown (O).

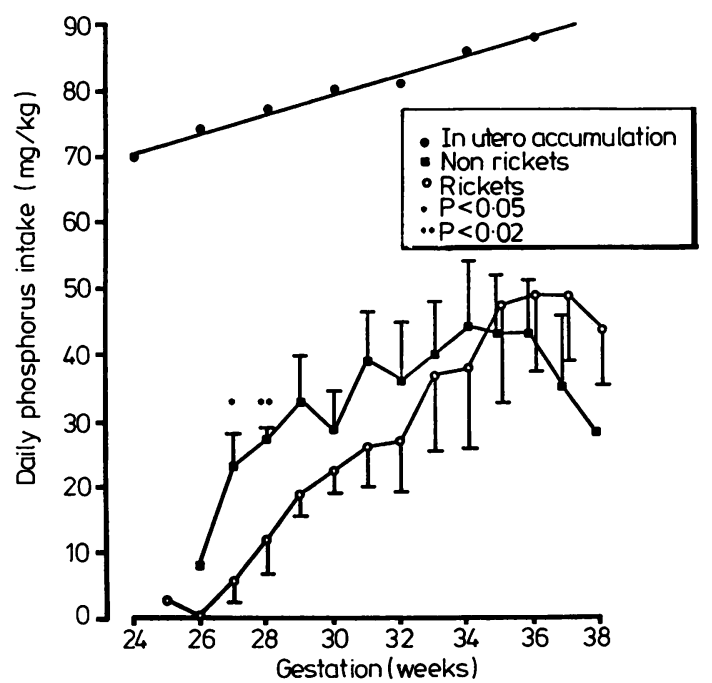

Fig. 2 Daily enteral phosphorus intake of 8 neonatal survivors of $<1000 \mathrm{~g}$ birthewight developing rickets (O) compared to 7 neonatal survivors of $<1000 \mathrm{~g}$ birthweight not developing rickets $(\square)$. The in utero accumulation rate of Shaw is also shown (O).

\section{References}

1 McIntosh N, Livesey A, Brooke OG. Plasma 25 hydroxyvitamin $D$ and rickets in infants of extremely low birthweight. Arch Dis Child 1982; 57: 848-50.
2 Day GM, Chance GW, Radde IC, Reilly BJ, Parks E, Sheepers J. Growth and mineral metabolism in very low birth weight infants. II Effects of calcium supplementation on growth and divalent cations. Pediatr Res 1975; 9: 568-75.

${ }^{3}$ Steichen JJ, Gratton TL, Tsang RC. Osteopenia of prematurity: the cause and possible treatment. $J$ Pediatr 1980; 96: 528-34.

4 Rowe JC, Wood DH, Rowe DW, Raisz LG. Nutritional hypophosphataemic rickets in a premature infant fed breast milk. New Engl J Med 1979; 300: 293-86.

5 Shaw JCL. Evidence for defective skeletal mineralization in low birth weight infants. The absorption of calcium and fats. Pediatrics 1976; 57: 16-25.

\section{Haemophilus influenzae infection in the newborn}

Sir,

In recent months we have encountered 2 newborn infants with congenital Haemophilus influenzae infection. The first was a 32 week preterm infant (birthweight $1.5 \mathrm{~kg}$ ) delivered vaginally, who developed respiratory symptoms shortly after birth. The chest $x$-ray film showed right lower lobe consolidation. $H$ influenzae was isolated from the blood culture, gastric aspirate, and superficial swabs. The infant made a satisfactory recovery on intravenous ampicillin and gentamicin.

The second infant was delivered vaginally at term (birthweight $2.8 \mathrm{~kg}$ ). She exhibited fetal distress during labour. She was acutely ill from birth, being tachypnoeic, acidotic, and shocked. $H$ influenzae type B was isolated from the blood cultures and gastric aspirate. Despite treatment with antibiotics, assisted ventilation, and peritoneal dialysis for actue renal failure she died at age 3 days.

Although we had not previously seen congenital $H$ influenzae infection at our unit, a series of 9 cases have been reported in the United States. ${ }^{1}$ The severity of the infection is emphasised by the high mortality in that report. After our recent experience we think that the possibility of $\boldsymbol{H}$ influenzae should be considered when selecting an antibiotic regimen for the infected newborn before obtaining culture reports. We would be interested to know if other units are experiencing problems with this organism.

\section{Reference}

1 Lilien LD, Yeh, TF, Novak GM, Jacobs NM. Early onset haemophilus sepsis in newborn infants: clinical, roentgenographic and pathologic features. Pediatrics 1978; 62: 299-303.

J F MURPhy AND P Minchom Department of Child Health, Welsh National School of Medicine, Heath Park, Cardiff CF4 $4 X N$ 